\title{
Estimating the Dynamic Ratio of the Lateral/Medial Hamstrings. A Case Control Study
}

\author{
N. Malliaropoulos ${ }^{1,2,3,6}$, G. Bikos ${ }^{4}$, D. Pyne ${ }^{2}$, G. Kakavas ${ }^{5}$, N. Maffulli3,7,8, P. Tsaklis 9,10 \\ 1 Sports and Exercise Medicine Clinic, Thessaloniki, Asklipiou, Greece \\ 2 Sports Clinic, Rheumatology Department, Barts Health NHS Trust, London, UK \\ 3 Centre for Sports and Exercise Medicine, Queen Mary, University of London, London, UK \\ 4 Euromedica-Arogi Rehabilitation Clinic, Pylaia, Thessaloniki, Greece \\ 5 Fysiotek Spine and Sports Lab Athens, Athens, Greece \\ 6 London Sports Care, BMI London Independent Hospital, London, UK \\ 7 Department of Orthopaedics, School of Medicine, Surgery and Dentistry, Salerno, Italy \\ 8 School of Pharmacy and Bioengineering, Keele University School of Medicine, Stoke on Trent, UK \\ 9 Biomechanics and Ergonomics, ErgoMech Lab, Department of Physical Education and Sport Science, University \\ of Thessaly, Trikala, Greece \\ ${ }^{10}$ Department of Molecular Medicine and Surgery, Growth and Metabolism, Karolinska Institutet, Sweden
}

\section{CORRESPONDING AUTHOR:}

Nicola Maffulli

Centre for Sports and Exercise Medicine

Queen Mary

University of London

Mile End Road

London, United Kingdom

E-mail: n.maffulli@qmul.ac.uk

DOI:

10.32098/mltj.02.2021.14

LEVEL OF EVIDENCE: 4

\begin{abstract}
SUMMARY
Purpose. Hamstring injuries are among the most common injuries in sports. The current study estimates the hamstrings dynamic ratio (HDR) between the mean muscle activation of lateral (biceps femoris longus) and medial part (semitendinosus) of the hamstring muscles during a maximal voluntary isometric contraction in elite uninjured track and field athletes.

Methods. Following isokinetic assessment to estimate the hamstrings to quadriceps dynamic ratio $(\mathrm{H}: \mathrm{Q})$, the participants were divided in two groups (Group $1(\mathrm{~N}=10)$ : athletes with a H:Q ratio range $55-70 \%$ and no side to side difference of the ratios more than 6 units; and Group $2(\mathrm{~N}=8)$ athletes with a $\mathrm{H}: \mathrm{Q}$ ratio outside of the $55-70 \%$ range and/or a side to side difference of the ratios greater than 6 units). All athletes were assessed by electromyography during a maximum voluntary isometric contraction of their hamstrings.

Results. There was difference on the range and means between the two groups regarding hamstrings dynamic ratio $(78 \%$ and $69 \%$ respectively), although this difference did not reach statistical significance.

Conclusions. A proposed HDR of $78 \%$ between lateral to medial hamstrings could be used as a risk factor for hamstring injuries in athletes with hamstring muscle imbalances. Better characterisation of the normal patterns of hamstring muscle activation will allow targeted rehabilitation to address specific neuromuscular coordination patterns.
\end{abstract}

\section{KEY WORDS}

Athletes; isokinetic testing; muscle injuries; track and field.

\section{INTRODUCTION}

The hamstring muscles comprise the biceps femoris muscle laterally and the semimembranosus and semitendinosus muscles medially. Crossing both the hip and knee joints, they produce both hip extension and knee flexion. Muscle strain injuries of this group are common in running based sports and are the most frequently reported injury in track and field (1). At the 2011 IAAF World Championships, hamstring strains were the most common injury, with $67 \%$ of them resulting in absence from sport. Increased repeated use of the hamstring was the predominant cause of injury (1) and approximately 
$80 \%$ of hamstring strains involve the long head of the biceps femoris (2).

A systematic review of hamstring muscle strain injuries in sport found the most consistent risk factors for this injury to be intrinsic factors such as age, previous hamstring injury and an increase in quadriceps peak torque (3-5). The available evidence for hamstring weakness as a risk factor for injury is conflicting: a recent meta-analysis did not support strength deficit as a risk factor (3), but the aetiology of hamstring injuries is multifactorial (6), and hamstring strengthening is an important component of injury prevention programmes (7). Isokinetic dynamometry is widely used in the assessment of the hamstring strength (8). In 614 soccer players (9) there was a small but significant association between lower hamstring eccentric strength and increased risk of injury. Using isokinetic strength testing, side to side differences in hamstring peak torque and decreased strength of the hamstrings to quadriceps, or H:Q ratio, have been suggested as risk factors for hamstring injury (10). Although it is difficult to generalise and no definite consensus exists, the normal $\mathrm{H}$ :Q ratio is considered to be $50 \%$ to $80 \%$ over the full range of knee motion (11). A side-to-side difference of $>10 \%$ is generally considered abnormal (12). In 6 sprinters with an acute hamstring injury, Sugiura et al. reported a significantly decreased eccentric and concentric peak torque in the injured vs non-injured limb and in H:Q ratio (13).

Electromyography (EMG) records and quantifies the electrical activity associated with contracting skeletal muscle fibres (14). Each of the hamstring muscles has different properties in terms of architecture (15) and contraction activation patterns on EMG, depending on whether they act as knee flexors (16) or hip extensors (17). Heterogeneous hamstring activation patterns are evident during different strength training exercises and during the gait cycle or sprinting (18), indicating complex neuromuscular coordination patterns. Previously injured hamstrings exhibit altered muscle activation patterns, with earlier EMG onsets for both the biceps femoris and medial hamstrings in preparation for single leg standing compared to the control group (19). In contrast, the rate of torque development and onset of muscle activity as measured by EMG is not associated with future hamstring injury (20). Using functional MRI, a more symmetrical recruitment pattern between the hamstring muscles has been shown in previously injured football players (21).

Excessive use, strength imbalances and altered muscle activation patterns have been suggested to contribute to the risk of hamstring injury (22). Thus, it is important to clarify more the role and kinetics of the medial and lateral hamstrings, known that they also act as medial and lateral rotators of the tibia respectively, during the swing face and stabilizers of the tibia during the stance face of gait and running. The optimal dynamic ratio of muscle activation between the medial and lateral hamstrings during contraction in the uninjured population with no strength imbalances is not known. The present investigation aims to estimate the hamstrings dynamic ratio (HDR) between the mean muscle activation of the lateral (biceps femoris longus, BFL) and medial (semitendinosus, ST) hamstrings during a maximal voluntary isometric contraction. This information may be used to prevent hamstrings unilateral overuse and secure their kinetic activation and function.

\section{MATERIALS AND METHODS}

Participant characteristics

Eighteen male elite track and field athletes (ten sprinters, five long jumpers, three triple jumpers; mean age $24.4 \pm$ 4.1 years; mean height of $177.4 \pm 6.5 \mathrm{~cm}$; mean body mass $78.8 \pm 5.6 \mathrm{~kg}$ ) from the Greek national team gave their written informed consent to participate in this study. None of them had reported a hamstring injury during the previous 6 months according to the clinic archives. Informed consent has been obtained from all individuals included in the study. The current study has been complied with the ethical standards of the journal (23) and all the relevant national regulations, institutional policies and in accordance the tenets of the Helsinki Declaration, and has been approved by the ethics committee of the Greek Track and Field Federation.

\section{Methodology}

The participants executed an isokinetic test to estimate the dynamic ratio between the Flexors and Extensors of the knee joint (H:Q ratio). Following that, an EMG of the biceps femoris longus and semitendinosus muscles during maximal voluntary isometric contraction (MVIC) was performed. The hamstrings dynamic ratio (HDR) was determined from the individuals who presented a isokinetic $\mathrm{H}$ : Q ratio within the range $55-70 \%$ and with a side to side difference in the ratios no greater than 6 units.

\section{Isokinetic test}

An isokinetic dynamometer (CSMI, Lumex, Ronkonkoma, NY, USA) was used. The participants completed a warm up of 10 minutes of submaximal cycling and 10 minutes of static stretching of the thigh muscles (quadriceps/hamstrings and adduction/abduction), preceded by familiarization with the isokinetic dynamometer with five submaximal repetitions performed at low, medium and high angular velocities $\left(90-120-180^{\circ} / \mathrm{sec}\right)$. The subjects were strapped in the dynamometer according to standard practice. The range 


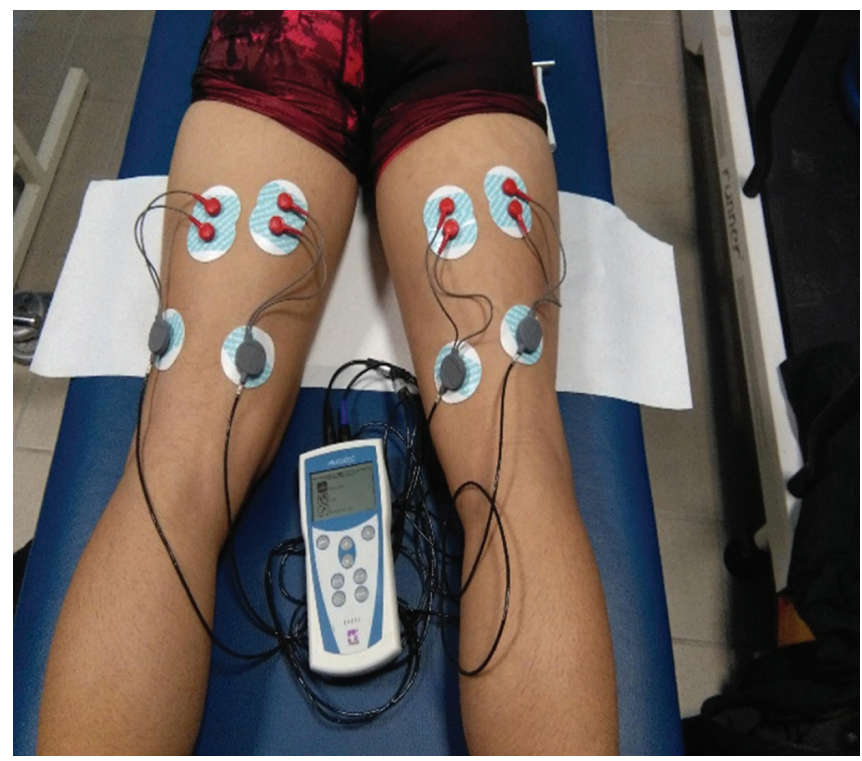

Figure 1. Electrodes placement.

of motion was set at $120^{\circ}\left(0^{\circ}\right.$ full extension). The angular velocity of both flexion and extension was set at $60^{\circ} / \mathrm{sec}$, for 5 repetitions at the highest possible effort. Visual (screen) and verbal (tester) feedback were used to encourage that each athlete performed at his maximum.

\section{EMG recording}

EMG recording was performed using the 8-channel Biomonitor ME6000 (Mega Electronics Ltd, Linton, UK). Prior to EMG recording, the skin of each participant was shaved, sand-papered, and carefully cleaned with $70 \%$ alcohol. The biceps femoris longus and semitendinosus muscles were sampled, as they are the most commonly affected muscles in case of injury to the posterior thigh muscles. Disposable pre-gelled self-adhesive bipolar surface electrodes $(\mathrm{Ag} / \mathrm{AgCl}, 0.8 \mathrm{~cm}$ in diameter, Blue Sensor N-00-S, Medicotest A/S, Ølstykke, Denmark) were placed on the leg with an inter-electrode distance of $2 \mathrm{~cm}$ in accordance with SENIAM (Surface ElectroMyoFigurey for the Non-Invasive Assessment of Muscle) guidelines (http://www.seniam.org) (figure 1). This protocol was used to measure the electrical activity of the relevant muscle during MVIC.

Each participant was given a detailed presentation of the procedure for the MVICs and allowed to familiarise with the isometric test on the isokinetic dynamometer. The participants were prone, with the knee stabilized at $45^{\circ}$ of flexion, with neutral rotation of the tibia. The subjects performed two or three isometric contractions at almost

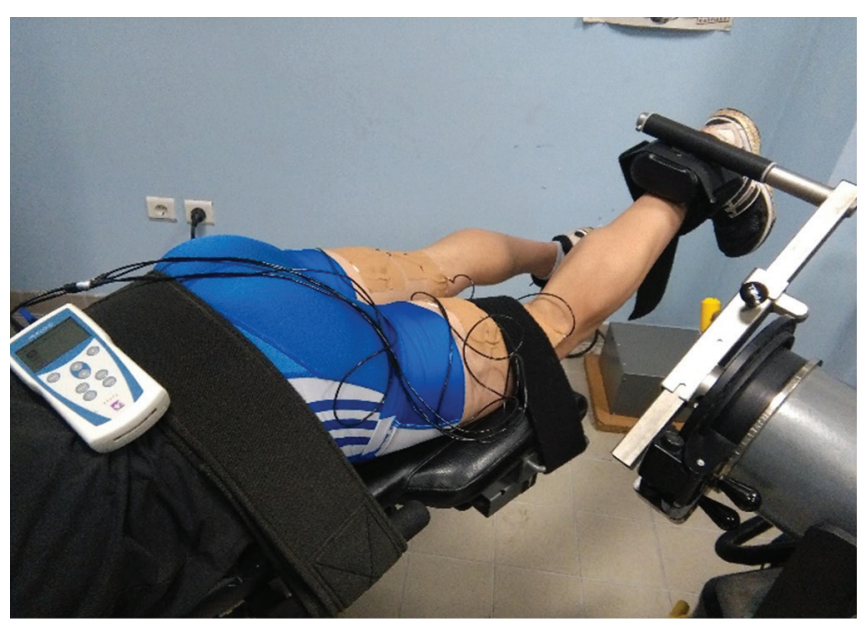

Figure 2. Maximal voluntary isometric contraction (MVIC) at the Isokinetic dynamometer.

$50 \%$ of maximum perceptual effort as a warm up. During the actual test, they performed three MVICs lasting five seconds each, with a five second rest between each contraction. The EMG signal $(\mu \mathrm{V})$ from the two recorded channels was averaged. Standardized verbal encouragement and visual feedback from the monitor was given to each participant. After a root-mean-square $(\mu \mathrm{V})$ adjustment, the trial with the highest averaged EMG signal was chosen, and then the $80 \%$ value of the last 3 seconds of this trial, was used as the final MVIC value (figure 2). The hamstrings dynamic ratio (HDR) between the BFL and ST muscles was calculated based on the BFLEMG/STEMG percentage value.

\section{Statistical analysis}

\section{Group separation for comparison analysis}

Based on the isokinetic $\mathrm{H}$ : Q ratio, the participants were separated in two groups: Group $1(\mathrm{~N}=10)$ : athletes with a $\mathrm{H}: \mathrm{Q}$ ratio range $55-70 \%$ and no side to side difference of the ratios more than 6 units; and Group $2(\mathrm{~N}=8)$ athletes with a $\mathrm{H}$ : Q ratio outside of the $55-70 \%$ range and/or a side to side difference of the ratios greater than 6 units (figures 3, $4 \mathrm{~A}$ ). Descriptive statistics were performed to calculate mean/ median/range/percentiles and SD of the (HDR) and Isokinetic $\mathrm{H}: \mathrm{Q}$ ratio, for each group. A paired samples t-test was also performed, between the groups, for each variable.

\section{RESULTS}

The athletes in Group 1 had a dynamic ratio of BFL/ST, based on the EMG, of $78.4(\mathrm{SD}=5.1)$. The mean BFL/ST 


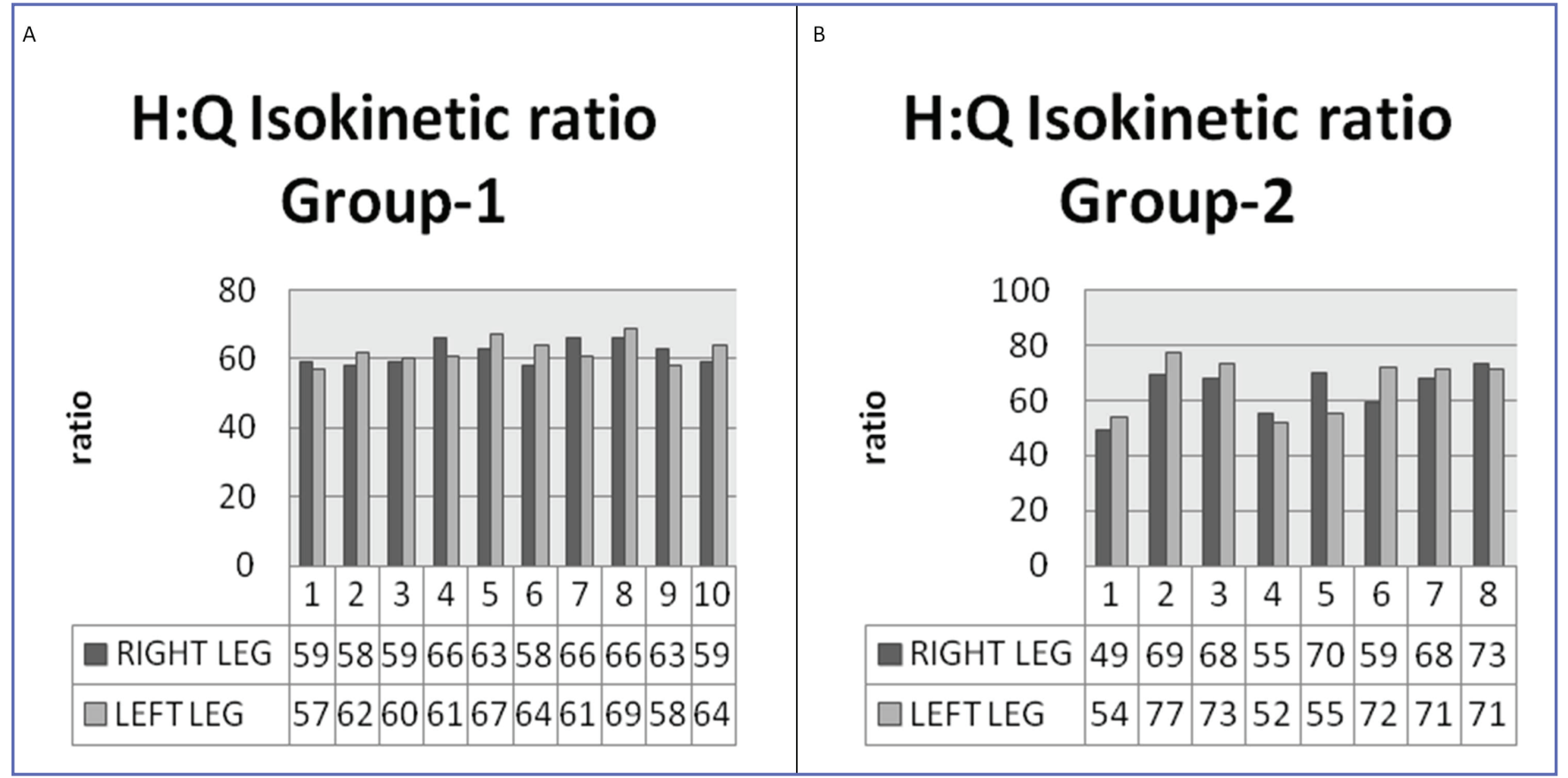

Figure 3. $\mathrm{H}: \mathrm{Q}$ Isokinetic ratio in Group 1 (A) and in Group 2 (B).

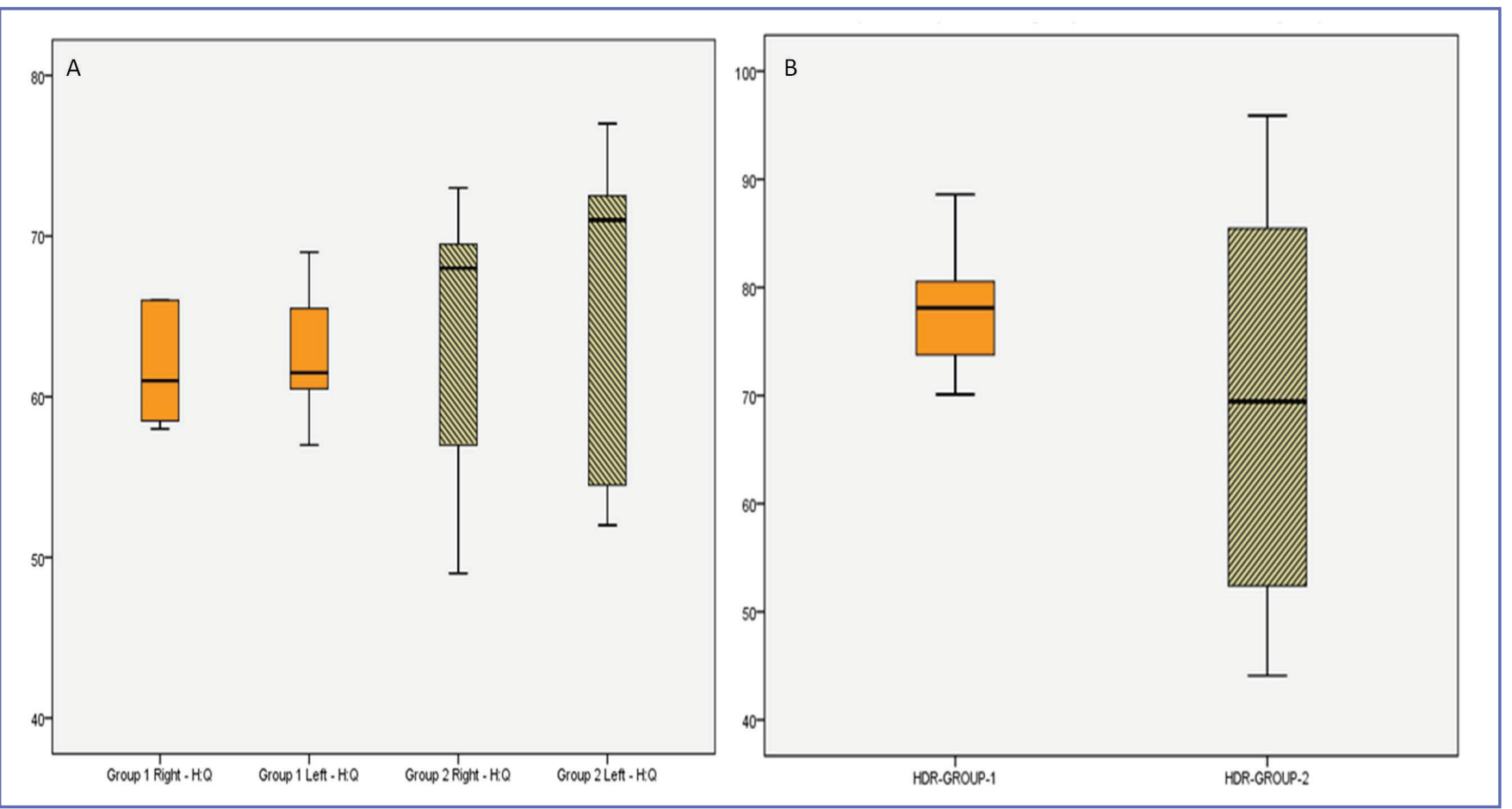

Figure 4. (A) Isokinetic H:Q ratio for the Right and Left leg between groups. (B) Hamstring dynamic ratio (HDR): medians and range between groups. 


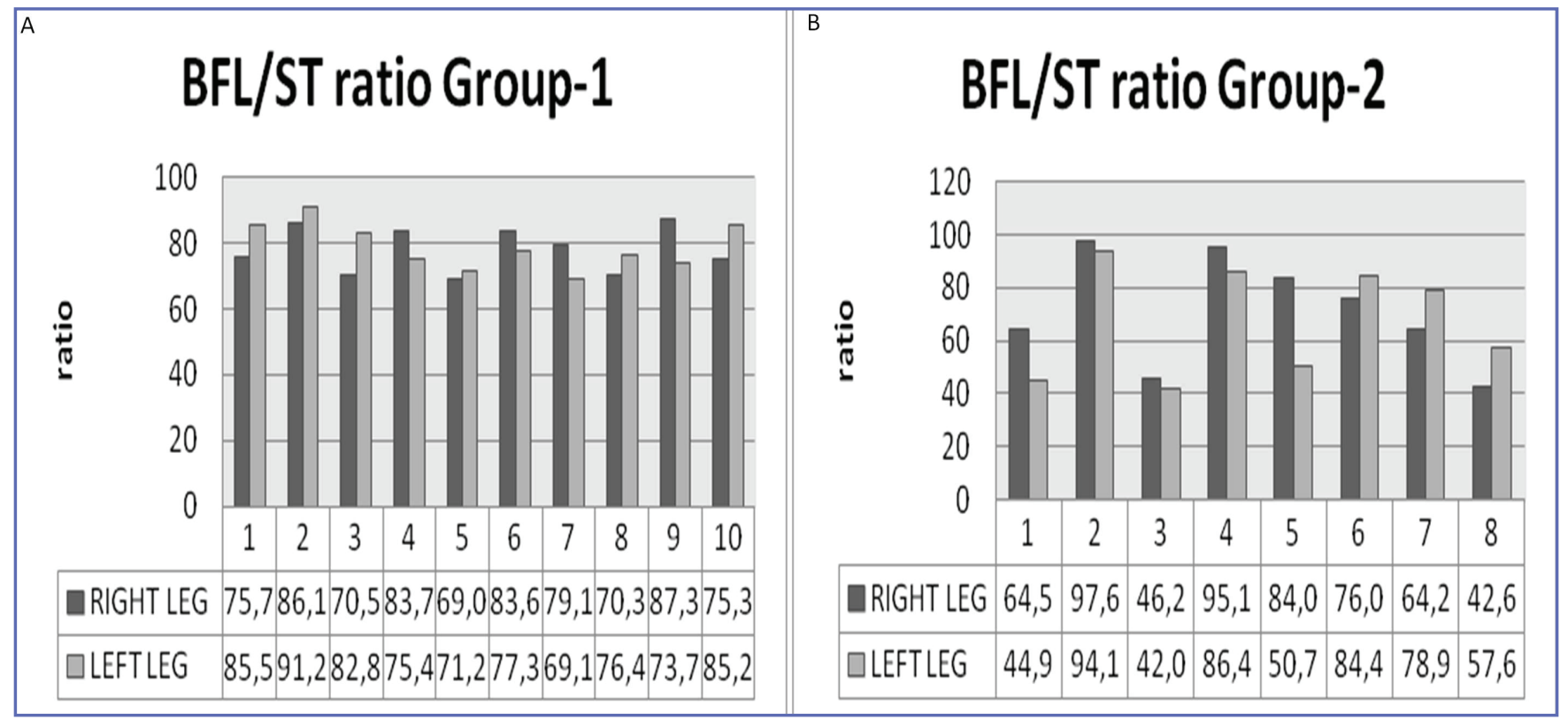

Figure 5. Hamstring dynamic ratio (HDR) means in Group 1 (A) and IN Group 2 (B).

ratio for the Group 2 was 69.3 ( $\mathrm{SD}=18.9)$ (figure 5). There were no statistically significant differences in the BFL/ST ratio between the groups ( $\mathrm{p}=.179)$. The values in Group 1 athletes showed less variation $(r=18.5)$, with a median of 79.9 compared with the values in Group 2 athletes $(\mathrm{r}=51.9)$ with a median of 69.3 (figure $4 \mathrm{~B}$ ). The results in Group 1, coupled with the specified bilaterally dynamic balance (H:Q ratio), show that the Hamstring dynamic ratio (HDR) between BFL and ST muscles is around $78 \%$ (figures $4 \mathrm{~B}, 5$ ).

\section{DISCUSSION}

The main result of the present study is that, in elite uninjured track and field athletes with H:Q ratios within the normal range and no side-to-side asymmetry, the HDR of $\mathrm{BFL} / \mathrm{ST}$ muscle activity during isometric hamstring contraction is around $78 \%$. Although not significantly different in this population of elite track and field athletes in the present investigation, the HDR in athletes with strength asymmetries or $\mathrm{H}: \mathrm{Q}$ ratios out of the normal range were lower, around $69 \%$.

These results are in part in keeping with the study by Scheurmans et al. (21), who used functional MRI to assess individual hamstring muscle activation during eccentric exercise. In that investigation, the biceps femoris and semitendinosus muscles engage in complex synergistic activation patterns during eccentric exercises, with more symmetrical degrees of activation in the injured group, together with higher overall levels of metabolic activity in the hamstring group. This would suggest that a more symmetrical muscle activation pattern during a hamstring task may imply a less efficient maladaptive compensatory mechanism.

\section{Practical implications}

The lateral to medial ratio difference in our population may imply a change in muscle activation patterns in those athletes possibly more prone to hamstring injury, as suggested by altered H:Q ratios and side to side strength asymmetries. Obviously, the design of the study does not allow to ascertain which factor is causative or reactive. However, the present study contributes to a greater understanding of the characters of hamstring muscles contraction in healthy athletes.

\section{CONCLUSIONS}

A proposed HDR of $78 \%$ between lateral to medial hamstrings could be used as a risk factor for hamstring injuries in athletes with hamstring muscle imbalances. Better characterisation of the normal patterns of hamstring muscle activation will allow targeted rehabilitation to address specific neuromuscular coordination patterns, using exercises that preferentially target individual muscles of the hamstring group. 


\section{Limitations}

In the present study, we did not investigate the pattern of activation of the semimembranosus muscle (SM), which acts together with the semitendinosus as a medial knee flexor. This was because of the difficulty to collect clear EMG signal from this muscle. We therefore assumed that the dynamic EMG outputs collected from the ST muscle are representative of the activity of the SM, given their analogous mechanical and physiological function.

We acknowledge that the current study was relatively small. However, they were all elite track and field athletes who regularly competed in international competitions up to world and Olympic level. In this respect, it would be difficult to recruit a larger population. We nevertheless acknowledge that further work to examine muscle activation during hamstring contractions with larger cohorts is needed. Additionally, recent literature (24) has shown different incidence rate and different hamstring injury distribution in younger athletic population, and therefore the results could be different in according to the age of the athletes. Another limitation is that the study entry criteria did not exclude other peripheral injuries in the ipsilateral lower extremity, which could be a factor of potential alterations in hamstring muscle activity (25).

In the future, it would be interesting to examine not only the amount of muscle activity but the timing of muscle activity as well. EMG would allow assessing the timing of muscle activation to be assessed, an advantage over functional MRI. Then, following athletes longitudinally would allow understanding whether altered synergistic relationship between the medial and lateral hamstrings on isokinetic testing is associated with injury development. However, such study

\section{REFERENCES}

1. Alonso JM, Edouard P, Fischetto G, Adams B, Depiesse F, Mountjoy M. Determination of future prevention strategies in elite track and field: analysis of Daegu 2011 IAAF Championships injuries and illnesses surveillance. Br J Sports Med 2012;46:505-14.

2. Opar DA, Williams MD, Timmins RG, Hickey J, Duhig SJ, Shield AJ. Eccentric hamstring strength and hamstring injury risk in Australian footballers. Med Sci Sports Exerc 2015;47:857-65.

3. Freckleton G, Pizzari T. Risk factors for hamstring muscle strain injury in sport: a systematic review and meta-analysis. $\mathrm{Br}$ J Sports Med 2013;47:351-8.

4. Valle X, Alentorn-Geli E, Tol JL, et al. Muscle Injuries in Sports: A New Evidence-Informed and Expert Consensus-Based Classification with Clinical Application. Sports Med 2017;47:1241-53. would necessitate large number of athletes, and relatively long periods of observation.

\section{CONTRIBUTIONS}

Nikolaos Malliaropoulos planned the study and contributed to write the manuscript. Panagiotis Tsaklis performed the data collection and statistical analysis. Georgios Bikos, Dev Pyne and George Kakavas contributed to write the manuscript. Nicola Mafulli contributed to interpret the data, and wrote and edited the manuscript. All authors have read and approved the final version of the manuscript, and agree with the order of presentation of the authors.

\section{ETHICS}

The ethics committee of the Greek Track and Field Federation approved the study.

\section{FUNDINGS}

No author has any financial interest or received any financial benefit from this research.

\section{ACKNOWLEDGMENTS}

The results of the current study do not constitute endorsement of the product by the authors or the journal.

\section{CONFLICT OF INTERESTS}

The authors declare that they have no conflict of interests.

5. Malliaropoulos N, Isinkaye T, Tsitas K, Maffulli N. Reinjury after acute posterior thigh muscle injuries in elite track and field athletes. Am J Sports Med 2011;39:304-10.

6. Malliaropoulos N, Papacostas E, Kiritsi O, Papalada A, Gougoulias N, Maffulli N. Posterior thigh muscle injuries in elite track and field athletes. Am J Sports Med 2010;38:1813-9.

7. Donaldson A, Cook J, Gabbe B, Lloyd DG, Young W, Finch CF. Bridging the gap between content and context: establishing expert consensus on the content of an exercise training program to prevent lower-limb injuries. Clin J Sport Med 2015;25:221-9.

8. Osternig LR. Isokinetic dynamometry: implications for muscle testing and rehabilitation. Exerc Sport Sci Rev 1986;14:45-80.

9. van Dyk N, Bahr R, Whiteley R, et al. Hamstring and Quadriceps Isokinetic Strength Deficits Are Weak Risk Factors for Hamstring Strain Injuries: A 4-Year Cohort Study. Am J Sports Med 2016;44:1789-95. 
10. Croisier JL, Ganteaume S, Binet J, Genty M, Ferret JM. Strength imbalances and prevention of hamstring injury in professional soccer players: a prospective study. Am J Sports Med 2008;36:1469-75.

11. Rosene JM, Fogarty TD, Mahaffey BL. Isokinetic Hamstrings:Quadriceps Ratios in Intercollegiate Athletes. J Athl Train 2001;36:378-83.

12. Daneshjoo A, Rahnama N, Mokhtar AH, Yusof A. Bilateral and unilateral asymmetries of isokinetic strength and flexibility in male young professional soccer players. J Hum Kinet 2013;36:45-53.

13. Sugiura Y, Saito T, Sakuraba K, Sakuma K, Suzuki E. Strength deficits identified with concentric action of the hip extensors and eccentric action of the hamstrings predispose to hamstring injury in elite sprinters. J Orthop Sports Phys Ther 2008;38:457-64.

14. Farina D, Merletti R, Enoka RM. The extraction of neural strategies from the surface EMG. J Appl Physiol (1985) 2004;96:1486-95.

15. Woodley SJ, Mercer SR. Hamstring muscles: architecture and innervation. Cells Tissues Organs 2005;179:125-41.

16. Onishi H, Yagi R, Oyama M, Akasaka K, Ihashi K, Handa Y. EMG-angle relationship of the hamstring muscles during maximum knee flexion. J Electromyogr Kinesiol 2002;12:399-406.

17. Ono T, Higashihara A, Fukubayashi T. Hamstring functions during hip-extension exercise assessed with electromyography and magnetic resonance imaging. Res Sports Med 2011;19:42-52.

18. Higashihara A, Ono T, Kubota J, Okuwaki T, Fukubayashi T. Functional differences in the activity of the hamstring muscles with increasing running speed. J Sports Sci 2010;28:1085-92.

19. Sole G, Milosavljevic S, Nicholson H, Sullivan SJ. Altered muscle activation following hamstring injuries. $\mathrm{Br} \mathrm{J}$ Sports Med 2012;46:118-23.

20. van Dyk N, Bahr R, Burnett AF, Verhagen E, von Tiggelen D, Witvrouw E. No association between rate of torque development and onset of muscle activity with increased risk of hamstring injury in elite football. Scand J Med Sci Sports 2018;28:2153-63.

21. Schuermans J, Van Tiggelen D, Danneels L, Witvrouw E. Biceps femoris and semitendinosus--teammates or competitors? New insights into hamstring injury mechanisms in male football players: a muscle functional MRI study. Br J Sports Med 2014;48:1599-606.

22. Tsaklis P, Malliaropoulos N, Mendiguchia J, et al. Muscle and intensity based hamstring exercise classification in elite female track and field athletes: implications for exercise selection during rehabilitation. Open Access J Sports Med 2015;6:209-17.

23. Padulo J, Oliva F, Frizziero A, Maffulli N. Muscles, Ligaments and Tendons Journal - Basic principles and recommendations in clinical and field Science Research: 2018 update. Muscles Ligaments Tendons J 2018;8(3):305-7.

24. Valle X, Malliaropoulos N, Parraga Botero JD, et al. Hamstring and other thigh injuries in children and young athletes. Scand J Med Sci Sports 2018;28:2630-7.

25. Malliaropoulos N, Bikos G, Meke M, et al. Higher frequency of hamstring injuries in elite track and field athletes who had a previous injury to the ankle - a 17 years observational cohort study. J Foot Ankle Res 2018;11:7. 